\title{
Urbaner Wandel und soziale Fragmentierung Einleitung zum Themenheft
}

\section{Gabriel Wackermann, Paris}

\section{Von Entwicklungsprozessen zur sozialen Fragestellung}

Jahrhundertelang war die Stadt «ein Ganzes». Sie hatte Aussenviertel («faubourgs»), die sich bis in die neuzeitliche Geschichte hinein jenseits der Stadtmauer entwickelten. Vom Mittelalter an über die Zeit des politischen Wandels im ausgehenden 18. Jh. und bis in die erste Hälfte des 19. Jh. wurden dort unerwünschte Bevölkerungsgruppen von der Stadt ferngehalten, manche oft nur nachts, so zum Beispiel Bettler, Obdachlose und wandernde Händler. Im Industriezeitalter entstanden ausserhalb der Stadt Bannmeilen, und die Viertel innerhalb der Stadt wurden sozialen Umschichtungen durch "Invasions- und Sukzessionsprozesse» ausgesetzt. Es entstanden sozialräumlich stark differenzierte Städte; sie liessen in den USA riesenhafte Stadtgebiete des sozialen und baulichen Verfalls und in europäischen Metropolen unter anderem die «roten» Stadtgürtel entstehen, die zur Herausforderung für die bürgerliche Gesellschaft wurden.

Das postindustrielle Zeitalter beschleunigte besonders seit dem Zweiten Weltkrieg den Umbau der Stadt zu einer «Stadtlandschaft». Suburbanisierung, Deurbanisierung und Reurbanisierung haben die ehemaligen Schranken zwischen Stadt und Umland verwischt (Cheshire \& Gornostaeva 2001, Wackermann 2000). Die Entwicklung der Metropol- zu Megalopolräumen unterzog auch die Problematik der Städte einem ausserordentlichen Wandel (Ascher 1995). In der zweiten Hälfte des 20. Jahrhunderts haben sich die meisten Grossstädte der Welt so schnell entwickelt, dass ihre heutige Immobiliensubstanz überwiegend kaum mehr als hundert Jahre alt ist. In Brüssel beispielsweise sind ungefähr zwei Drittel der Wohnhäuser und des Stadtraumes erst nach 1900 entstanden, in Paris rund 80 Prozent. In Metropolen der Entwicklungsländer ist das Alter der Bausubstanz weit geringer: In Teheran z.B. sind Privathäuser, die mehr als ein halbes Jahrhundert alt sind, eine Seltenheit.

Urbaner und suburbaner Raum unterlagen zugleich einer intensiven Konzentration und einer internationalen Öffnung in Richtung Handel und Dienstleistungen (NiTsCH 2001). Die Globalisierung und die daraus hervorgegangene Beschleunigung der Vernetzungsmechanismen (BLOTEVOGEL, OSSENBRÜGGE, WOOD
2000) führten zu Agglomerationsräumen und schliesslich zu Metropolregionen mit sich ergänzenden oder gegenüberstehenden Teilräumen. Die Agglomeration als solche behielt zwar ihre morphologische und funktionelle Bedeutung, hing jedoch in immer stärkerem Masse von den eigenen Standortwerten und quantitativen sowie qualitativen Raumergänzungsangeboten des erweiterten Umlands ab (WACKERMANN 2002). Das durch morphologische Kontinuität und hohe Bevölkerungsdichte abgegrenzte Agglomerationskonzept erlaubt es nicht mehr, die wirkliche Metropolitanrolle der Agglomeration zu definieren. Die vielfältigen Agglomerations-Umlandvernetzungen und die wechselseitigen sozialen und wirtschaftlichen Einflüsse führen zu neuen Ansätzen, den gesamten Regionalraum und die Impulskraft des Zentralpols zu erfassen (Geographica Helvetica 3/2001, Kruse 2001).

Statistisch genügt es derzeit nicht mehr, mit elementaren statischen, wenn auch international vergleichbaren Daten zu operieren. Die ausserordentliche Vielfalt und Spezialisierung hochrangiger Dienstleistungen, die nur im Rahmen der vernetzten Metropolregion ihre Optimalentfaltung erreichen können (GEENHUIZEN 2001), entgehen oft der offiziellen Statistik. Dies ist ganz besonders der Fall, wenn es sich um die Metafunktionen Technologieentwicklung und Innovation handelt (Behrendt \& Kruse 2001). Daher sind Agglomerationsabgrenzungen wie jene, die seit 1989 vom N.U.R.E.C. (Network on Urban Research in the European Union) für die Europäische Union erarbeitet wurden, für eine eingehende dynamische Metropolforschung - soziale Fragmentierung einbegriffen - nur noch Ansatzpunkte. Selbst das Konzept der europäischen Metropolregion ergibt, «dass die Einführung der Metropolen-Semantik in die deutsche Raumordnungspolitik als Reaktion auf eine ganze Reihe von externen Herausforderungen zu verstehen ist» (Blotevogel 2001). Die Metropolregion ist ein Gesamtes, welches als Entscheidungs-, Kontroll- und Koordinationszentrum von internationaler Bedeutung erscheint. Der mangelnde wissenschaftliche Abstand erlaubt jedoch noch nicht, festzustellen, inwiefern sogar in einer Metropolregion wie Frankfurt/RheinMain diese gebündelte Dreierfunktion gesichert ist (Schamp 2001).

Obwohl die urprüngliche Stadt als prestigeträchtiges Aushängeschild oft zur Innenstadt, City, Touristenund Freizeitstadt, und somit gewissermassen zur Museumsstadt reduziert wurde, erlebte sie gleichzeitig sozi- 
alen Niedergang, baulichen Verfall und Ghettoisierung. Dies führte zwar zu Aufwertungsmassnahmen durch Gentrifikation und "gated communities», was jedoch auch Nachteile mit sich bringt. Die Innenstadt, in denen Megaprojekte der Stadtentwicklung realisiert werden, verkörpert heutzutage nur noch einen winzigen Teil der Kernstadt einer Metropolagglomeration, die insgesamt von hohen sozialen und finanziellen Kosten herausgefordert wird. Sozialräumliche Fragmentierung, Armut und Sozialhilfeabhängigkeit sowie deren kulturelle Folgen nehmen rasch zu (FARWICK 2001). Der Verlust des Augenmasses für den Menschen in der Stadt und die Masslosigkeit im Städtebau sind dabei miteinander verbunden. Der Raum wird auf Kosten Dritter, u.a. Steuerzahler und nachkommender Generationen, erschöpft. Der Leitsatz «Stadt macht frei» gehört der Vergangenheit an, und die komplexen Konfliktsituationen im Stadtraum zeigen sich auch international in einer allgemeinen Krise der Stadt.

Man kann natürlich eine Stadtmetropole als Konsequenz und Manifestation des Fortschritts werten, so wie man auch die Bedeutung eines Staates selbst eines Entwicklungslandes - in Kenngrössen von Bevölkerung oder Wirtschaft messen kann. Weder Bevölkerungsgrösse noch wirtschaftliche Dynamik bewirken jedoch per se «Urbanität» und eine optimale Stadtenwicklung. Die dynamischsten Agglomerationen weisen beispielsweise oft recht hohe Arbeitslosenquoten auf. Daher ist es angebracht, die sozialen Folgen der Verstädterung und Metropolisierung eingehend in Blick auf die Entwicklungsdynamik von Kernstadt und suburbanem Raum zu untersuchen und Segregation sowie sozialen Verfall bzw. soziale Fragmentierung als eine der wichtigsten Problemstellungen unserer Gesellschaften zu thematisieren (SCHNEIDER-SLIWA 1996).

Die Dimension der aktuellen Stadtentwicklung, städtische Sozialpathologien inbegriffen, führt wieder dazu, den Menschen als Mass der Dinge und die Lebensbedingungen in Städten ins Zentrum der wissenschaftlichen Betrachtung zu rücken, wobei diese in der Geographie auch als Resultat einer global-lokalen Dialektik thematisiert werden (SCHNEIDER-SLIwA 2002). Allerdings bedarf es noch vielfacher empirischer Arbeiten, um aktuelle soziale Realitäten im Stadtraum, die sich immer kleinräumiger abzeichnen, analytisch und kartographisch zu erfassen.

\section{Die Verstädterung als Zukunftsproblem: Die Suche nach einem Sinn der Stadt}

Die Krise, in welcher sich die urbanisierte Welt befindet, ist in einen allgemeinen Krisenzyklus eingebunden, der zusammen mit der Komplexität der Stadt die Möglichkeit des friedlichen Miteinanderlebens immer mehr in Frage stellt, es ist dies eine «Komplexität, die als positiven Effekt das Entstehen eines Solidaritätsgefühls auf Weltebene hervorrufen kann» (ReEves 1996): Von der Entropie ausgehend zur Negentropie könnte sich dadurch das Stadtsystem neu aufbauen.

Seit der Industriezeit wurde die Stadt funktionell nach und nach zerrissen. Persönlichkeiten wie Haussmann und LE CORBusier und dessen «Schule», die sich dem Credo der Charta von Athen zuwandten, haben dazu beigetragen, diese Tendenz mit ihrem Fokus auf Architektur vor Stadtentwicklung als Ganzem zu verstärken. Die ausserordentlichen Umwälzungen, die aus dem Industriezeitalter und der postfordistischen Epoche resultieren, stellen zwingend die Frage einer Ethik und dadurch auch einer Ästhetik, die dem Fortschritt angepasst ist und zugleich dem Grundbedürfnis der Menschen als Individuen und Gemeinschaftswesen entspricht. Die meisten urbanen sozialen Fragestellungen sind auf tiefe Zeit- und Raumkontinuitätsbrüche zurückzuführen sowie auf die Diskrepanz zwischen Lokalem und Globalem. Die wirtschaftliche Entwicklung vernachlässigte kulturelle Eigenheiten und somit viele kulturelle Werte.

In den sechziger Jahren schreibt der französische Soziologe Henri Lefèbvre, dass «bisher nur die Dichter die Stadt als Heim des Menschen verstanden haben». Mit Emotion und Sensibilität betrachtet LEwIS Mumford bereits Jahre früher die Stadt als ein Meisterwerk der Zivilisation. Er lässt sich durch das Gartenstadtmodell von EBENEZER Howard beeinflussen. Die «new towns» sollen zu neuer Inspiration und kultureller Rückkehr anspornen. In seinem Buch «Skyline-La ville Narcisse» (Die Narzistische Stadt) weist Hubert Damisch sogar auf die kulturelle Rolle des Wolkenkratzers hin: «Die Stadt erzeugt den Wolkenkratzer, der Wolkenkratzer erzeugt jedoch nicht die Stadt» (Damisch 1996). Ausserdem zeichnet sich die Postmetropolis durch Widersprüchlichkeit aus, einerseits weist sie sphinxhaft auf viele Möglichkeiten hin, andererseits ist sie klar durch die Wissensökonomie als besonderem Qualitätsfaktor strukturiert, die «den metropolitanen Regionalstandort als Denkraum kennzeichnet» (HELBRECHT 2001).

Die Pluri- und besonders die Interdisziplinarität haben zudem zur Neubesinnung auf die Stadt in Forschung und Praxis geführt. Die Suche nach dem Sinn der derzeitigen Stadt und der Verstädterung sowie das erneuerte Stadtdenken (GHORRA-Gobin 2001) haben zur Notwendigkeit angepasster sozialpolitischer Massnahmen geführt. Beispiele sind die Dekonzentrierung und die damit verbundenen erweiterten Vernetzungen des urbanen Raumes, die Meisterung von Flächennutzungsproblemen, die Festlegung einer annehmbaren Mobilität für die Agglomerations- und 
Metropolräume, eine den ökologischen Gegebenheiten angeglichene Wirtschaftsattraktivität sowie die Erforschung räumlicher Potentiale und angemessener Siedlungsentwicklung (KREIBICH 1999). Nachhaltige Entwicklungsstrategien benötigen sowohl eine den Struktur- und Konjunkturverhältnissen angepasste koordinierte Wirtschaftslogik als auch ein entsprechendes Sozialverhalten.

Die angestrebten Neuorientierungen sind jedoch nicht effizient genug, wenn man nicht vorher grundsätzliche Fragen stellt und dazu innovative Lösungsansätze zu finden sucht (Siebel 2000). Für Walter Siebel bleibt es zweifelhaft, ob man immer noch die Stadt als Standort der wirtschaftlichen Emanzipation betrachten kann. Er erwägt, ob es nicht angebrachter ist, sich auf eine demokratische Selbstverwaltung zu stützen und wirft die Fragen nach den Voraussetzungen auf, mit denen die Stadt als Standort sozialer Integration gestaltet werden kann. Ob dies in der traditionellen europäischen Stadt überhaupt noch möglich ist, lässt sich letzten Endes auch für andere Städte weltweit diskutieren.

\section{Die Beiträge und deren Aussagekraft}

Die in diesem Themenheft veröffentlichten Aufsätze geben Anlass dazu, die durch den urbanen Wandel weltweit verursachte soziale Fragmentierung unter verschiedenen Grundaspekten zu analysieren und dementsprechende Lösungsansätze ausfindig zu machen, die nicht nur lokale Aussagekraft, sondern generelle Anwendbarkeit haben. Die ausgewählten Fallbeispiele sind für theoretische Überlegungen besonders angebracht.

SUSANNE Eder untersucht eingehend am Metropolbeispiel Basel-Stadt die residentiellen Segregationsmuster, welche sich aus den städtischen Sozialstrukturen entwickeln. Sie geht von den neueren gesamtgesellschaftlichen Strukturveränderungen aus, um die aufgrund von Einwanderungen eingetretene Heterogenisierung zu analysieren, wobei festgestellt wird, dass sich kleinräumig soziale Milieus gebildet haben und eine Mosaikstruktur besteht. Unter dem Einfluss der Wohnumfeldqualität entwickeln sich Abgrenzungsund Konzentrationsprozesse, die innerhalb der Stadt ein neues soziales Profil mit starker Heterogenität der Sozialgruppen bilden.

ERNST STEINICKE stellt mit einer tiefgreifenden Analyse am Beispiel der slowenischen ethnischen Minderheiten in Kärnten fest, dass auch in der postfordistischen Epoche in urbanen Räumen autochthone, ethnolinguistische, nicht dominierende Volksgruppen vor dem Assimilationsprozess bewahrt und gefördert werden können. Unter der Voraussetzung eines rechtlichen Minderheitenschutzes ist es innovativen Kräften möglich, ethnische Rückbesinnung in ihrem gesellschaftlichen Milieu anzustreben. Zentralörtliche Einrichtungen wie Gymnasien tragen dazu bei, Akteure auszubilden, die die Selbstbehauptung der Minderheitsgruppe nicht nur im städtischen Raum, sondern auch im Umfeld stärken und regionale Ethnodiversität entfalten, eine Entwicklung, die als ein Reichtum im derzeitigen regionalen Wettbewerb erscheint.

Joachim Burdack und Robert Rudolph zeigen, dass sich auch die Städte der postsozialistischen Welt den Erfordernissen der globalen Wirtschaft so gut wie möglich anpassen, obwohl der Intensitätsgrad dieser Integration von Stadt zu Stadt sehr verschieden ist. Die strukturellen Hemmnisse sind qualitativ und quantitativ noch zahlreich, die eigenen Lösungswege ebenfalls. Die gutinformierten Autoren analysieren diese Entwicklung anhand des Vergleichs von vier typischen Städten: Moskau, Budapest, Jekaterinburg und Łódź. Trotz der starken Ausdifferenzierung verbleiben osteuropäische Städte von übergreifenden Transformationsphänomenen gekennzeichnet.

Der Aufsatz von Martin Coy gibt einen Einblick in die urbane und metropolitanregionale Problematik der Entwicklungsländer. Die Untersuchung stützt sich auf den derzeitigen Stand der Metropolitanregionen Brasiliens, wo das rasche städtische Wachstum die soziale Segregation dadurch verstärkt hat, dass sich zugleich Marginalviertel ausdehnen und privilegierte Wohnghettos entstehen. Der Verfall der Innenstädte lässt die Zentrumsfunktionen zum Stadtrand hin entlang der neuen Entwicklungsachsen entstehen. Die gemeinsame Aufgabe der Stadtverwaltungen und der NichtRegierungsorganisationen besteht in dem Versuch, die Stadtzentren zu revitalisieren.

Das Problem der Suche nach einer regionalen Stadt - auf Europa bezogen - wirft WiLliam SaLET auf. Er weist darauf hin, dass Initiativen des privaten Sektors in die Raumentwicklungspolitik einbezogen werden müssen, was eine Neuverteilung der Rollen auf zentraler Ebene erforderlich macht. Dieser Schritt wird jedoch nicht immer gemacht. Er stellt fest, dass die Prinzipien «local accountability» und «electoralism» im Gleichgewicht in die Praxis umgesetzt werden müssen, damit die grossstädtisch-regionale Dynamik das soziale und politische Gleichgewicht nicht gefährdet. Daher wird es unumgänglich sein, dass sich Bürger und Politiker nicht länger mit verblassten Zentrumsstädten identifizieren, sondern «neue regionale Identitäten mobilisieren».

\section{Literatur}

Ascher, F. (1995): Métapolis ou l'avenir des villes. Paris: Odile Jacob, 346 S. 
Behrendt, H. \& C. Kruse (2001) : Die Europäische Metropolregion Zürich - die Entstehung des subpolitischen Raumes. - In: Geographica Helvetica 3: 202-213.

Blotevogel, H.H. (2001): Die Metropolregionen in der Raumordnungspolitik Deutschlands - ein neues strategisches Raumbild? - In: Geographica Helvetica 3: $157-168$.

Blotevogel, H.H., Ossenbrügge, J. \& G. Wood (Hrsg.) (2000): Lokal verankert - weltweit vernetzt. - In: Tagungsbericht und wissenschaftliche Abhandlungen, 52. Deutscher Geographentag Hamburg, Stuttgart: Franz Steiner Verlag.

Cheshire, P.C. \& G. Gornostaeva (2001): More useful Londons: The comparative development of alternative concepts of London. - In: Geographica Helvetica 3: 179-192.

Damisch, H. (1996): Skyline-La ville Narcisse. - Paris: Le Seuil.

Farwick, A. 2001: Armut in der Stadt - Prozesse der Ausweitung und Verfestigung räumlich segregierter Armut am Beispiel der Stadt Bielefeld. - In: Geographica Helvetica 2: 90-106.

GeEnHUizen VAN, M. (2001): Amsterdam as a changing node in financial services. - In: Geographica Helvetica 3: 193-201.

Ghorra-Gobin, C. (Hrsg.) (2001) : Réinventer le sens de la ville: les espaces publics à l'heure globale. - Paris: L'Harmattan, $268 \mathrm{~S}$.

Helbrecht, I. (2001): Postmetropolis: Die Stadt als Sphinx. - In: Geographica Helvetica 3: 193-201.

Kreibich, V. (1999): Operationalisierung einer zukunftsfähigen Stadtentwicklung. - In: Hallesches Jahrbuch für Geowissenschaften, Reihe A, Geographie und Geoökologie 21, Halle: 27-41.

KruSE, C. (2001): Funktionale Räume und europäische Metropolregionen: Analyse, Empirie und politische Handlungsebenen - Einführung in das Themenheft. In: Geographica Helvetica 3: 154-156.

Nitsch, V. (2001) : City growth in Europe. - = Volkswirtschaftliche Schriften 518, Duncker \& Humblot, Berlin, $185 \mathrm{~S}$.
Reeves, H. (1996): L'avenir de la complexité. - In : Liberté 226, Paris: 6-8.

Schamp, E.W. (2001): Der Aufstieg von Frankfurt/ Rhein-Main zur europäischen Metropolregion. - In: Geographica Helvetica 3: 169-178.

SchNeIDER-Sliwa, R. (Hrsg.) (2002): Städte im Umbruch - Globalisierung und Geographie in der Neustrukturierung von Berlin, Brüssel, Hanoi, Ho-ChiMinh-Stadt, Hongkong, Jerusalem, Johannesburg, Moskau, Sankt-Petersburg, Sarajewo und Wien - Berlin: Reimer Verlag, 350 S. (im Druck).

SCHNEIDER-Sliwa, R. (1996): Kernstadtverfall und Modelle der Erneuerung in den USA. Privatism, public-private partnerships, Revitalisierungspolitik und sozialräumliche Prozesse in Atlanta, Boston und Washington D.C. - Berlin: D. Reimer Verlag, 353 S.

Siebel, W. (2000): Die Zukunft der europäischen Urbanität. - In: Nachrichten, 2, Akademie für Raumforschung und Landesplanung, Hannover: 39.

WACKERMANN, G. (2002): Géographie régionale. - Paris: Ellipses, 250 S. (im Druck).

WACKERMANN, G. (2000): Très grandes villes et métropolisation. - Paris: Ellipses, $223 \mathrm{~S}$.

Prof. Dr. em. Gabriel Wackermann (Paris-Sorbonne IV)

180, Route d'Oberhausbergen, F-67200 Strasbourg. e-mail: OB88278033@aol.com 